\title{
Raman spectroscopy in biology and medicine
}

\begin{abstract}
The mini review focuses on the expected applications of Raman spectroscopy in biology and medicine. The possibility of in vivo and in situ measurements will contribute to the development of biology and will generate the necessary knowledge required to understand biological processes. It is concluded that Raman spectroscopy will become a very strong endoscopic technique based on laser diodes with excitation wavelengths ranging from UV to NIR. Few example that demonstrate the strength of Raman spectroscopy in biology and medical fields are briefly discussed.
\end{abstract}

Keywords: Raman spectroscopy, Raman endoscopy, Therapeutic window
Volume 3 Issue 4 - 2016

\author{
Dorina Magdalena C,' Elamin Ibrahim, ${ }^{2}$ Luis \\ Materon, ${ }^{3}$ Desiree Padilla, ${ }^{3}$ Mircea Chipara' \\ 'Department of Physics and Astronomy, The University of Texas \\ Rio Grande Valley, USA \\ ${ }^{2}$ Department of Chemistry, The University of Texas Rio Grande \\ Valley, USA \\ ${ }^{3}$ Department of Biology, The University of Texas Rio Grande \\ Valley
}

Correspondence: Mircea Chipara, Department of Physics and Astronomy, The University of Texas Rio Grande Valley, $1201 \mathrm{~W}$. University Drive, Edinburg, TX 78539, USA Tel: | 956605 5I23 Email chipara@yahoo.com

Received: February 01, 2016 | Published: April 23, 2016

\section{Introduction}

It is expected that the future development of biology and medicine will be dominated by a massive transfer of experimental techniques now available to physicists and chemists. This will provide to biologists and medical scientists information at atomic and molecular scales, accelerating the knowledge in these rapidly growing areas of science.

Among the experimental techniques expected to dominate the biological and medical research during the next decade, Raman spectroscopy is expected to play an important role. The advantages of Raman spectroscopy derives from its simplicity and versatility. Technically, the method does not require a special preparation of the sample, can be easily adapted for in situ/in vivo or endoscopic measurements. Compared to FTIR, the Raman spectroscopy is significantly less affected by the presence of water and $\mathrm{CO}_{2}$. Fluorescence is a big problem for Raman spectroscopy. The simplest method to decrease the fluorescence is to use a laser with a wavelength as high as possible. For polymeric materials $785 \mathrm{~nm}$ is one of the most frequently used wavelength. For biological tissues, there is a window of electromagnetic radiation ranging between 650 and 1350 $\mathrm{cm}^{-1}$, characterized by a weak absorption of the incoming radiation. This optical or therapeutic window will be exploited by future Raman system designed for bio and medical applications.

\section{A simple and quick guide to Raman spectroscopy}

Raman spectroscopy senses motions (vibrations and eventually rotations) at atomic and molecular scale. The basic principle of Raman spectroscopy implies the excitation of atoms and molecules by an incident beam of electromagnetic radiation (of a given frequency, $\mathrm{n})$, followed by the analysis of the energy released by the sample at all frequencies excepting the frequency of the incoming beam (n) during the de-excitation process. From the physicist's point of view, the Raman Effect reflects the inelastic collision of electromagnetic radiation with the sample (matter). It is not required for the frequency of the incoming electromagnetic radiation to correspond to an energy level of the sample. If this occurs, then the intensity of the Raman Effect is increased by several orders of magnitude (this is also known as Resonant Raman). FTIR is a complementary technique that investigates also atomic and molecular vibrations. By contrast, in
FTIR the incoming electromagnetic radiation is swept over a given frequency range and the amount of energy absorbed by the sample from the incoming electromagnetic radiation is recorded. This corresponds to elastic collisions between the incoming electromagnetic radiation and the sample and represents formally a resonant excitation from one energy level to a higher one. As expected, the Raman signal is several orders of magnitude weaker than the intensity of FTIR lines. Usually, both Raman and FTIR spectra are ranging from $100 \mathrm{~cm}^{-1}$ up to about $4000 \mathrm{~cm}^{-1}$. FTIR spectra reflect the change in the dipole moment of the sample while Raman spectra indicate a change of the polarizability of the sample. Consequently,

A Raman system includes a source of electromagnetic radiation (usually a laser diode that can eventually emit a polarized laser beam), an optical system to guide the laser beam onto the sample and to collect the reflected beam, a dispersive element necessary to analyze the received electromagnetic radiation upon frequencies (or wave numbers), and a detector (frequently a CCD camera cooled down by a thermoelectric element). Portable Raman spectrometers or stationary Raman systems that offer the capability to extract the beam by using optical cables are commercially available. There are no apparent restrictions to biological applications of Raman spectroscopy. For medical applications, care is required to select a laser diode that is not producing damages while providing sufficient power to record Raman spectra with an acceptable signal to noise ratio.

\section{Raman spectroscopy in biology and medicine}

Existing Raman units can be easily adapted for medical needs, especially if the laser diode exploits the therapeutic window. Unfortunately, even in this frequency range, the incoming laser beam penetrates only a shallow region. Accordingly, most medical applications of Raman spectroscopy are expected from refinements of endoscopic techniques, where the decrease of the optical cables diameter and improved flexibility will open the window to complex optical endoscopic systems that involves the usual investigation in the visible light and in situ Raman capabilities. The system may include local microscopy as well as local laser surgery capabilities. Such system may operate in a wide range of wavelengths from UV to NIR providing substantially improved diagnostic capabilities.

Raman in biology and medicine: The Beginning. 


\section{Acknowledgements}

All contributing authors (Dorina Magdalena Chipara, Elamin Ibrahim, Luis Materon, Desiree Padilla, Mircea Chipara*) have equal contributions to this manuscript.

\section{Conflicts of interest}

None. 